\title{
On the Rainbow, a Robert Grosseteste's Treatise on Optics
}

\section{Amelia Carolina Sparavigna'}

\author{
${ }^{1}$ Department of Applied Science and Technology, Politecnico di Torino, Torino, Italy
}

\begin{abstract}
On the Rainbow is one of the short scientific treatises written by Robert Grosseteste. The Latin title is De Iride. In the first part of this treatise, we can find a discussion on reflection and refraction of light, described in the framework of the geometrical optics. In the second part, Grosseteste is writing about the rainbow and how its colors are created.
\end{abstract}

Keywords: History of Science, Medieval Science, Optics.

\section{Introduction}

Robert Grosseteste (c.1175-1253) was an English scientist and philosopher of the Middle Ages. Born into an Anglo-Norman family in the county of Suffolk in England, he became Bishop of Lincoln from $1235 \mathrm{AD}$. He is considered one of the most prominent and remarkable figures of the thirteenthcentury [1], a thinker that played a key role in the development of scientific methods, as remarked by several scholars [2-4]. One of them, A.C. Crombie, even claimed Grosseteste as the first in the Latin West to develop an account of an experimental method in science, with his systematic use of the method of "experimental verification and falsification" [1,3]. However, it is necessary to tell that Grosseteste's experimental method was quite different from the modern methods used in controlled experiments. Grosseteste in fact derived his conclusions on the basis of a mix of considerations, appealing to authority and everyday observation (the Latin "experimentum"). He made use of thought experiments and some certain metaphysical assumptions, such as the principle of "least action", that we will find here, in reading the De Iride, On the Rainbow, one of his scientific treatises.

In the next section, I am proposing a translation of De Iride. In spite of its title, the treatise is not only about the rainbow. In the first part of the text in fact, we can find a discussion of reflection and refraction of light. Besides these phenomena that Grosseteste discussed also in his treatise entitled On Lines, Angles and Figures [5], we have some words about optical instruments too. In the second part of De Iride, Grosseteste continues writing about the rainbow as a phenomenon of refraction of light. He explains how the shape of the rainbow is originated and the creation of its colors. The original Latin text used for the translation is in Reference 6 .

\section{On the Rainbow}

Optics and physics have to speculate on the rainbow. However, the same "what" the physics needs to know, is a "because of what" the optics needs. And in fact, Aristotle, in the book on the meteorology, did not show "because of what", in the sense of optics, but "what" is the rainbow, which is physics, in a quite short discussion. Hence, here, in this paper, the "because of what" concerning optics is started discussing and explaining in our manner and time opportunity.

First then, let us say that optics is a science based on the figures of the visual perceptions, and it is subaltern to the science based upon figures and schemes, which contains lines and radiating surfaces, being them cast by the radiating sun, or by stars, or by any other radiant body. And it has not to be thought that the going out of visual rays from eyes is only a virtual argument, without any reality, as people, who consider "the part and not the whole", are arguing. But let us note that visible objects are of a nature similar to the nature of the shining and sparkling sun, the radiation of which, combined with the radiation of the external surface of a body, completes the total perspective of vision.

Therefore, some philosophers, handling these natural things, are considering the natural visual perception as passive, that is, as an "intro-mission". However, mathematicians and physicists, concerning the nature of visual perception, consider that it occurs according to an "out-emission". Now, this part of the sight, which is effected by an out-emission, Aristotle plainly discussed in the last chapter of his book on the animals, that "the back of the eye sees far away; 
from its emission it is not divided, nor consumed, but its ability of sight goes forward from him and right to the things we are seeing." And again, in the same: "Three are our conscious senses, namely, sight, hearing and smell; they come out from the organs, just as water emerges from canals, and therefore a long nose has a good smelling." In optics, then, the true position concerning the rays is that of their emission.

Of which (optics), there are three main parts, according to the three ways of transition the rays have to the objects of vision. Either the path of the rays to the visible object is straight through a transparent medium having a specific feature, interposed between who is looking and the object. Or, it is ruled by a path directed to a body having a virtual nature, that is, a mirror, reflected by it, back to the object we are seeing. Or it is the passage of the rays through more transparent media of different kinds, where, at the interfaces, the ray is broken and makes an angle, and the ray comes to the object not with a straight path, but by means of several straight lines, having a number of angles at the related interfaces.

The first part of this science is named "de visu", the second "about mirrors". The third part is coming in our possession unknown and untouched. We know, however, that Aristotle had discussed this third part, which is the much more difficult, and the subtlety of which was by far the more remarkable, emerging from the deep heart of Nature. This part of optics, if fully understood, shows us the way in which we can made objects at very long distance appear at very close distance, and large things, closely situated, appear very small, and small things at a certain distance we can see as large as we want, so that, it is possible for us to read the smallest letters at incredible distance, or count the sand, or grain, or grass, or anything else so minute. In what way, however, it is necessary to understand how this wonder happens, so it will become clear to everybody.

Visual rays, penetrating through several transparent different materials, are broken at interfaces; and the parts of these rays, in the different existing transparent materials, at the interface of those are angularly connected. This, however, is clear by means of an experience, the principle of it is set down in the book on the mirrors: if we cast an object into a vessel, and the distance is assumed that it may not be seen, and some water poured into, it will be seen what is inside (see Figure 1).

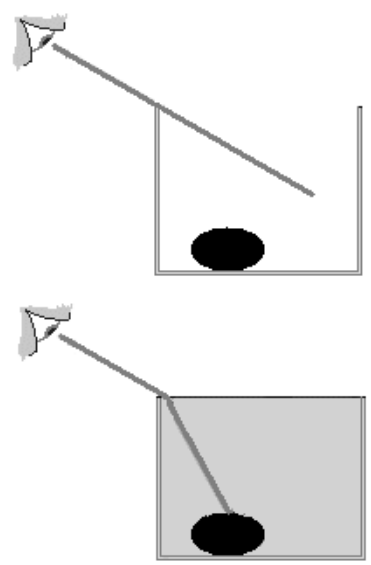

Figure 1: The experiment with the vessel.

And the same is displayed by a body having a continuous nature too; therefore, the visual ray, at the interface of two transparent media with different features, is subjected to a contiguity law. When one total ray is generated from a source, the continuity of it cannot be broken, except when its generation is broken, and at the interface of two transparent media, the ray cannot be discontinuous; at the interface, we cannot have a full continuity and a complete discontinuity and therefore, at each point of the interface the two parts of the ray are, not directly, but angularly connected.

But, how large is the angular deviation from the straight path associated to a ray? Let us consider the ray from the eye through the air medium, incident on a second transparent medium, as a straight line to the point, where it is incident on the transparent medium; then let us make the line deep in the transparent medium, line that makes equal angles with the surface of transparent medium, that is, normal to the interface. I say, therefore, that the prolongation of the ray in the second transparent medium is following a line, separating of a certain angle, which is one half of the angle " $i$ " obtained as follow. "i" is the angle given by the line which is the prolongation of the ray, without interruption and direct, drawn away from the point of incidence deep into the medium, equal to the angle " $i$ ", drawn above the surface of the second transparent medium. So we have determined the amount of the refractive angle of the rays. We know that there are similar experiments giving the refraction of the rays on mirrors, fitting an angle equal to the angle of incidence (Figure 2). And the same tells us that principle of the philosophy of Nature, namely, that "every action of the Nature is well established, most ordinate, and in the best and shortest manner as it is possible." 


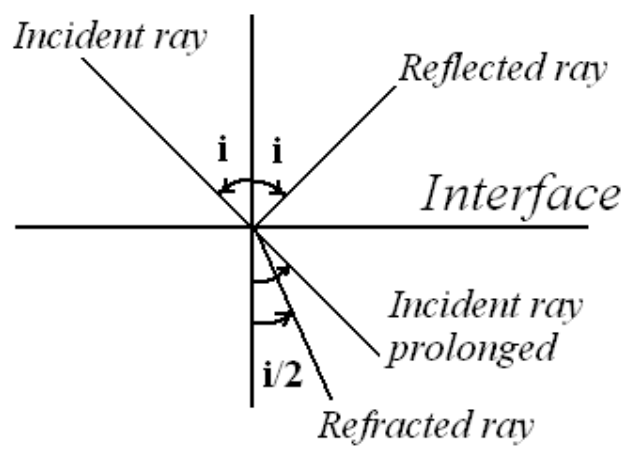

Figure 2: Grosseteste's law of reflection and refraction.

Moreover, the object which is seen through a medium composed of several transparent materials, does not appear to be as truly is, but it is appearing composed by the concurrence of the rays from the eye, continuous and direct, and by the lines starting from the viewed object and falling on the following surface, the nearest to the eye, according to its normal. This is clear to us from experiments and similar reasoning that we know: that an object seen in a mirror appears in the concurrence of the propagation of the lines of sight and the lines drawn directly upon the surface of the mirror, normal to this surface.

It is evident then what is the quantity of the angle according to which the ray is broken at the interface between transparent media and where the image of an object appears arising from several transparent media. Let us add also those principles of optics, which are given by the philosophers studying the natural phenomena, then we have the following: given the amount of the angle under which an object is seen, it appears its position and size, according to the order and organization of the rays. It is not the great distance rendering a thing invisible, except by accident, but the smallness of the angle under which it is seen. It is clear that it is possible, using geometrical ratios, knowing the position and the distance of the transparent medium, and knowing the distance from the eye, to tell how an object appears; that is, given its distance and size, it is possible to know the position and the size of the image. It is also clear how we can design the shape of the transparent medium, in order to have this medium able to receive the rays coming out from the eye, according to the angle we choose, collecting and focusing the rays as we like over the observed objects, whether they are large or small, or everywhere they are, at long or short distances. In such a way, all objects are visible, in the position and of the size given by the device; and large objects can appear short as we want, and those very short and at a far distance, on the other hand, appear quite large and very perceptible.
And in the third part of optics we have the study of the rainbow. Undoubtedly, it is not possible the rainbow be given by a direct crossing of the solar rays in the cavities of the clouds. Because the continuous illumination of the cloud does not produce an arc-like image, but some openings towards the sun, through which the rays enter the cavity of the cloud. And it is not possible that the rainbow is produced by a reflection of the rays of the sun upon the surfaces of the raindrops falling down from the cloud, as reflected by a convex mirror, so that the cavity of the cloud receives in this manner the reflected rays, because, if it would be so, the rainbow would not be an arc-like object; moreover, it would happen that increasing the altitude of the sun, the rainbow would be greater and higher, and decreasing the sun altitude, the rainbow would be smaller; this is contrary to what is shown by the experience. It is therefore necessary that the rainbow is created by the refraction of the sun's rays by the humidity carried by the cloud. Let me tell then, that outside the cloud is convex and inside it is hollow. This is clear from the nature of "light matter" and "heavy matter". And that, what we see of a cloud is smaller than a hemisphere, even though it appears to us as a hemisphere, and when the humidity comes down from inside of the cloud, it is necessary that it assumes the volume of a convex pyramid at the top, descending to the ground, and therefore it is condensed in the proximity of the earth, more than in its upper part.

Then, there are four transparent media overall, through which the rays of the sun penetrate, that is, pure air containing the cloud, second the cloud itself, third the highest and most rarefied humidity coming from the cloud, and fourth, the lower and denser part of that humidity. From all the things discussed before on refraction and related angles at the interface between two media, it is necessary the rays of the sun are first refracted at the boundary of air and cloud, and then at the boundary of cloud and humidity, so that, after these refractions, the rays are conveyed in the bulk of humidity, and after, they are broken again though its pyramidal cone, however, not assuming the shape of a round pyramid, but in the form similar to the curved surface of a round pyramid, expanded opposite to the sun. Therefore its shape is that of a bow, and to us (in England), the rainbow can be austral, and, because the aforesaid cone is close to the earth, and it is expanding opposite the sun, it is necessary that more than a half of that cone falls below the surface of the earth, and the rest of it falls on the cloud, opposite the sun. Accordingly, on sunrise or sunset, a semicircular rainbow appears and is larger; when the sun is in other positions, the rainbow appears as a portion of 
the semicircle. And, when the altitude of the sun is increasing, the portion of the rainbow decreases. And for this reason, in those places where the sun can reach the zenith, the rainbow never appears at noon.

Aristotle tells that the "quantity" of the different arcs we can see on sunrise and sunset is small, but, Aristotle's small "quantity" is to be understood not concerning the "size" but the luminosity, which happens because the rays are passing, during these hours, through a large quantity of vapor, much larger than in other hours of the day. Aristotle himself suggests as a consequence, that there is a reduction of that which shines because of the rays of the sun in the clouds.

For what concerns the colours of rainbows, let us remember that color is light mixed with a transparent medium; the medium is diversified according to the purity and impurity, and the light is fourfold divided; it is to be divided according to the brightness, and of course, to the obscurity, and according to intensity and tenuity; and according to these six different enumerations the variety of all the colors is generated, the variety of colors that appears in the different parts of a single rainbow, is mainly due to the intensity or tenuity of the rays of sun. Where there is a greater intensity of light, it appears that the colors are more luminous and bright: but where there is less intensity of light, it appears that the color turns to the dark color of Hyacinthus. And because the intensity of light and the decrease of intensity is not subjected to a rule, except in the case of light shining on a mirror, or passing through a transparent medium, which, by means of its own shape, can gathers the light in a certain place, and, in a certain place can disrupt the light, diminishing it, and the arrangement of receiving the light is not a fixed one, it is clear that that it is not in the skill of an artist to reproduce the rainbow, but it is possible to imitate accordingly to a certain arrangement.

On the other hand, the difference of the colors of a rainbow from those of other rainbows is due to the purity and impurity of the transparent medium supporting it, as well as from the brightness and obscurity of the light impressing it. If we have a pure transparent medium and bright light, the color is whitish. If the recipient medium is a mixture of vapors and mist and the light is hazy, as occurs near the East and West, the colors are less splendid and their brightness reduced. In the same manner, according to the enumeration of brightness and obscurity of light and of purity and impurity of the medium, all the arcs of various colors can be seen.

Here is the end of the discussion on the rainbow, according to a Lincolnian.

\section{Discussion and conclusion}

First of all, Grosseteste is distinguishing optics from physics. The physics is the description of natural phenomena, whereas optics (perspectiva ars, in Latin [7]) is analysing the reasons of the phenomena. Of course, optics is linked with the visual perception: about it, there were two ancient Greek schools, providing a different explanation of vision. The first was proposing an "emission theory": vision occurs by means of rays emanated from the eyes and received by objects. We can see an object directly, or by means of refracted rays, which come out of the eyes, move in a transparent medium and, after refraction, arrive to the object. Among the others, Euclid and Ptolemy followed this theory. The second school proposed the "intro-mission" approach that sees vision as coming from something, representative of the object, which is entering the eyes. Aristotle and Galen followed this theory, which seems to have some contact with modern theories [8]. In the Grosseteste's treatise, it seems that he had mixed Aristotle's ideas with the outemission theory, and therefore, in the translation I used simply "emission".

In the first part of the treatise on the raibow, Grosseteste is describing some phenomena that we can obtain with lenses; he seems to describe, for instance, a magnifying glass useful to see the small things or read the small letters in a book. Moreover, he tells that we can made things at very long distance appear at very close distance, and large things appear very small, and small things we can see as large as we want. Had he some sort of microscope or telescope? May be; in any case, we can suppose that he had some reading stones. A reading stone was a lens having hemispherical shape, that was placed on a text to magnify the letters, so that people with presbyopia could read. Reading stones were among the earliest common uses of lenses. According to Wikipedia [9], they were developed in the 8th century, by Abbas Ibn Firnas. The function of reading stones was replaced by the use of spectacles from the late 13th century onwards. Early reading stones were made from rock crystal (quartz) as well as glass.

To tell the true, the earliest written records of lenses date to Ancient Greece. In his play, The Clouds (424 $\mathrm{BCE}$ ), Aristophanes is mentioning a burning-glass, a lens used to focus the sun's rays to produce fire. Pliny the Elder show that burning-glasses were known to Romans [10], and mentions what was probably a corrective lens: Nero was said to watch the gladiatorial games using an emerald, probably concave to correct for myopia [11]. Pliny is also describing the magnifying effect of a glass globe filled with water. What is interesting in the 
Grosseteste description is that he find and remark the reason of these effects in the refractions of the rays.

Grosseteste is also proposing a law of refraction. This law is telling that the angle of refraction is onehalf the angle of incidence i. Of course, it is quite different from the Snell's law that we use, containing the trigonometric functions of angles and the refractive indices.

Long before Grosseteste, reflection and fraction of light had been studied by ancient Greek scientists. The fact that the reflected angle is equal to the incident angle was well known. However, refraction is a more complex phenomenon. Ptolemy found a relationship regarding the angles of refraction [12]; this was an empirical law, fitting figures with experimental data. He measured the refraction from air to water, and water to glass. Ptolemy plotted r, the refractive angle, against $i$, the incident angle, at ten-degree intervals from $i=0$ to $i=80$ degrees. The resulting values of $r$ were in agreement with the sinelaw. Alhazen, in his Book of Optics (1021), studied the refraction too. Refraction was accurately described by Ibn Sahl, of Baghdad, in the manuscript On Burning Mirrors and Lenses (984) [13]. He made use of it to work out the shapes of lenses that focus light with no geometric aberrations [13]. The law was rediscovered by Thomas Harriot in 1602, who did not publish his results although. In 1621, Willebrord Snellius (Snell) derived a mathematically equivalent form, that remained unpublished, during his life. René Descartes independently derived the law in terms of sines in 1637, in his treatise "Discourse on Method". After Descartes' solution, Pierre de Fermat proposed the same solution based on his principle of least time, postulating that "light travels between two given points along the path of shortest time." [14] Let us note that, in this treatise on the Rainbow, after a sentence on the reflection of rays from mirrors, Grosseteste writes a principle of "least action" too, quite before Fermat.

It is remarkable that Grosseteste does not use in any of his treatise on optics a term such as "diopter" or "dioptron" (instrument to look through), a term which is coming from Greek. The ancient Greek dioptra were astronomical and surveying instrument, dating from the 3 rd century BCE. The dioptra were sighting tubes or, alternatively, rods with a sight at both ends, attached to a stand. So, the ancient dioptra usually had not lenses. However, in Italian, we use "diottro", to define the interface between two different optical media. And "diottrica" is the science concerning the light refracted by diaphanous media. In English, the term diopter arrived from French, having the same meaning it has in Italian. Probably Grosseteste knew that the Greek term diopter was used for surveying; the second sense, that of optical medium, had not yet arrived from French.

After the part of the treatise on geometrical optics, where Grosseteste is telling that knowing the rules followed by the rays of light we can give the position and magnitude of the images of objects, he continues with the description of the rainbow. His theory on the rainbow, such the ideas of other medieval scholars on it [15], are partially coming from the ancient Greek and Roman science. For instance, Pliny the Elder is describing it as follow [16]: "what we name rainbows frequently occur, and are not considered either wonderful or ominous; for they do not predict, with certainty, either rain or fair weather. It is obvious, that the rays of the sun being projected upon a hollow cloud, and the light is thrown back to the sun and is refracted, and that the variety of colours is produced by a mixture of clouds, air, and fire. The rainbow is certainly never produced except in the part opposite to the sun, nor even in any other form except that of a semicircle. Nor are they ever formed at night, although Aristotle asserts that they are sometimes seen at that time; he acknowledges, however, that it can only be on the 14th day of the moon. They are seen in the winter the most frequently, when the days are shortening, after the autumnal equinox. They are not seen when the days increase again, after the vernal equinox, nor on the longest days, about the summer solstice, but frequently at the winter solstice, when the days are the shortest. When the sun is low they are high, and when the sun is high they are low; they are smaller when in the east or west, but are spread out wider; in the south they are small, but of a greater span. In the summer they are not seen at noon, but after the autumnal equinox at any hour: there are never more than two seen at once."

Pliny does not talk about the colours of the rainbow, which are instead discussed by Grosseteste, who continues the analysis of the nature of colours in another treatise entitled De Colore, which is very short, and probably of the mid-1220s [17]. In both De Iride and De Colore, Grosseteste tells that the colours are created by the purity or impurity of the transparent medium when light, intense or not, is passing through it. From ancient times, it was well known that a prism can create the color of the rainbow [18]. However, during the Middle Ages, it was believed they were produced by impurities in the medium; this idea survived until the Newton's experiments with prisms and his theory of the dispersion of light.

After reading this treatise, we can conclude stressing again what we told in the introduction. Undoubtedly, Grosseteste saw a key role for geometry in the 
explanation of natural phenomena.

Deeply concerned with a detailed investigation of Nature, his treatises were a strong stimulus to the thinkers in the Oxford of the fourteenth-century to start the progress towards the mathematical physics.

\section{References}

[1] N. Lewis, Robert Grosseteste, The Stanford Encyclopedia of Philosophy, Winter 2010 Edition, http://plato.stanford.edu/entries/grosseteste/

[2] G. ten Doesschate, Oxford and the revival of Optics in the thirteenth century, Vision Rev., Volume 1, 1962, Pages 313-342.

[3] A.C. Crombie, Grosseteste's Position in the History of Science, in Robert Grosseteste: Scholar and Bishop, ed. Daniel A. Callus, Oxford: Clarendon Press, 1955, Pages 98-120.

[4] Ludwig Baur, editor, Die Philosophischen Werke des Robert Grosseteste, Bischofs von Lincoln, Beiträge zur Geschichte der Philosophie des Mittelalters, Volume 9, 1912, Münster: Aschendorff Verlag. This volume is considered the standard edition of Grosseteste's philosophical works and scientific opuscula.

[5] A.C. Sparavigna, Robert Grosseteste and his Treatise on Lines, Angles and Figures in the Reflection and Refraction of Light, The
International Journal of Sciences, Volume 2, Issue September, 2013, in print

[6] The Latin text is that given by "The Electronic Grosseteste", http://www.grosseteste.com, which is reporting the printed source from Ref.4, Pages 72-78.

[7] D. Harper, Online Etymology Dictionary, 2012, http://www.etymonline.com/index.php

[8] http://en.wikipedia.org/wiki/Visual_perception

[9] http://en.wikipedia.org/wiki/Reading_stone

[10] Pliny the Elder, The Natural History, translated by John Bostock, XXXVII, Chap. 10.

[11] Pliny the Elder, The Natural History, translated by John Bostock, XXXVII, Chap. 16.

[12] http://en.wikipedia.org/wiki/Snell's_law

[13] A. Mark Smith, Ptolemy and the Foundations of Ancient Mathematical Optics: A Source Based Guided Study, American Philosophical Society, Volume 89, Part 3, 1999.

[14] http://en.wikipedia.org/wiki/Principle_of_least_action

[15] R.L. Lee Jr. and A.B. Fraser, The Rainbow Bridge: Rainbows in Art, Myth, and Science, Penn State Press, 2001.

[16] Pliny the Elder, The Natural History, translated by John Bostock, II, Chap.60.

[17] A.C. Sparavigna, Robert Grosseteste's colours, arXiv:1212.6336 [physics.hist-ph], arXiv, 2012.

[18] A.C. Sparavigna, The play of colours of prisms, arXiv:1207.3504 [physics.hist-ph], arXiv, 2012. 